\title{
Discapacidad en Colombia: un reto en la educación superior inclusiva
}

\section{Disability in Colombia: Challenges for Inclusive Higher Education}

\author{
Gloria Isabel Bermúdez Jaimes* \\ María Fernanda Bravo Delgado** \\ Diana Rocío Vargas Pineda ${ }^{* * *}$
}

Fecha de Recepción: Marzo 3 de 2009

Fecha de Aceptación: Julio 28 de 2010

\section{Resumen}

La inclusión de las personas con discapacidad en el sistema educativo de Colombia y más específicamente en la formación superior, se constituye en un desafio para las Instituciones. Desde el plano regional hasta el plano local se logran identificar elementos de similitud en los procesos y en los avances. Asimismo, se identifican falencias y requerimientos estructurales que condicionan el ingreso, permanencia y egreso de la población con discapacidad a procesos de formación en educación superior.

Desde esta perspectiva, el presente documento articula la reflexión académica sobre el estado actual de las prácticas inclusivas en Educación Superior en Colombia, estableciendo elementos que permitan vislumbrar los desafíos a los que ésta se encuentra abocada para lograr su propósito de inclusión social de las personas con discapacidad.

Palabras clave: inclusión educativa, educación superior inclusiva, discapacidad.

\section{Abstract}

The inclusion in the Colombian educative system of people with disabilities and specially at the higher level is a challenge for the Institutions. Form the regional level to local level, is able to recognize elements of similarity in the processes and progress. It also identifies gaps and structural requirements that determine the entry, stay and exit of people with disabilities in higher education.

\footnotetext{
* Fonoaudióloga, Magíster en Discapacidad e Inclusión Social, Universidad Nacional de Colombia. Miembro del Grupo de Investigación en Oralidad, escritura y Otros Lenguajes. Departamento de Comunicación Humana. Facultad de Medicina. Universidad Nacional de Colombia.gibermudezj@unal.edu.co

** Psicóloga, Magíster en Discapacidad e Inclusión Social. Universidad Nacional de Colombia. mfbravod@ unal.edu.co

*** Terapeuta Ocupacional, Magíster en Salud Pública. Universidad Nacional de Colombia. dianavargasp@ gmail.com
} 
From this perspective, this paper articulates the academic reflection on the current state of inclusive practices in Higher Education in Colombia, providing evidence to discern the challenges that it has been striving to achieve its purpose of social inclusion of people with disability.

Key words: inclusive education, inclusive higher education, disability.

\section{Introducción}

En Colombia, durante los últimos años se ha desatado un proceso de reflexión sobre la Educación Superior Inclusiva (ESI) que ha convocado a expertos, académicos, políticos y asociaciones de y para personas con discapacidad en la construcción de una plataforma política que favorezca la garantía del derecho a la educación para esta población. En este panorama las Instituciones de Educación Superior (IES) han jugado un papel esencial, no sólo por iniciar la reflexión académica sino además porque se han esforzado en abrir sus puertas al reto de la inclusión educativa de estudiantes con discapacidad con el ánimo de avanzar en la construcción de una sociedad incluyente.

Desde este escenario, el presente artículo propone una reflexión académica sobre el estado actual de las prácticas inclusivas en Educación Superior en Colombia, con el propósito de identificar los alcances y limitaciones de las mismas, y a partir de allí, proyectar los desafíos a los que éstas deben enfrentarse para lograr su propósito de contribuir a la inclusión social de las personas con discapacidad.

Esta reflexión inicia con una breve alusión al origen del concepto de inclusión educativa y sus desarrollos en América Latina, posteriormente, ofrece un recuento de los principales avances de la ESI en el contexto nacional y, finalmente, identificar los desafíos que debe encarar el país para alcanzar el propósito de garantizar la equiparación de oportunidades de las personas con discapacidad en la educación superior.

\section{La Inclusión Educativa y el Derecho a la Educación}

En el año 2001, como parte de un proceso de cambio en la concepción de la discapacidad que predominaba hasta el momento, la cual respondía al modelo médico-individual (1), la Organización Mundial de la Salud publicó la Clasificación Internacional del Funcionamiento, la Discapacidad y la Salud (2) en la que transformó la idea de que la discapacidad es un atributo del individuo y propuso una nueva concepción que la asume como un producto de la interacción entre el individuo y el entorno. Desde esta perspectiva, la discapacidad está determinada por las limitaciones en la actividad y las restricciones en la participación que un entorno no adaptado les impone a los individuos que presentan una deficiencia.

Esta nueva visión de la discapacidad, centrada en la idea de que los ambientes son los que deben adaptarse a los individuos, implicó la exigencia de introducir modificaciones significativas a todos los ámbitos en los que se desenvuelven las personas con discapacidad, incluido por supuesto, el contexto educativo. Así las cosas, garantizar el pleno derecho a la educación a las personas con discapacidad, implica, al igual que con el resto de la población, cumplir con los requisitos de accesibilidad, asequibilidad, adaptabilidad y aceptabilidad propuestas por Tomasevsky en su Informe sobre el Disfrute del Derecho a la educación en Colombia (3). Es decir, requiere la disposición de espacios suficientes, la gratuidad de la educación, la garantía de la no discriminación social, asegurar la permanencia del estudiante de comienzo a fin en el ciclo académico, y el desarrollo de sus capacidades. 
La inclusión educativa aparece entonces como una forma de materialización del derecho a la educación para las personas con discapacidad (4), que implica el desarrollo de procesos políticos, administrativos, sociales y culturales que permitan aumentar la participación de los estudiantes con discapacidad en la cultura, los currículos y las comunidades educativas (5).

Sobre este particular, como lo reiteran diversos autores (6) (7) (8) (9), la mayoría de los países de América Latina han dado más visibilidad a los grupos pobres, mujeres, población afro e indígenas, en comparación con la población con discapacidad. Las universidades han debatido sobre las cuotas en el ingreso para aspirantes provenientes de escuelas públicas, afro-descendientes e indígenas, dejando apartado del debate el caso de las personas con discapacidad, por ende, manteniendo hacia ellas dinámicas de exclusión.

Esta situación se hace preocupante si se considera la educación como un elemento transformador de las sociedades y se resalta que en la educación superior "su importancia estratégica se hace visible tan pronto como se reconocen los efectos de la incorporación de la ciencia y la tecnología, y de la reflexión elaborada sobre los fines, en los procesos de trabajo, en la producción de la riqueza material y simbólica y en el desarrollo de la organización social" (10).

El concepto de inclusión educativa se deriva de un amplio rango de políticas y conceptualizaciones internacionales que dieron inició la reflexión sobre las necesidades educativas de la población con discapacidad desde la perspectiva de la integración, noción que con el pasar del tiempo fue perdiendo vigencia en tanto que demostró mantener la lógica del modelo médico-individual que buscaba la normalización del individuo y no la de su entorno (4), pero que en su momento construyó la puerta de entrada al movimiento mundial aún vigente, que busca garantizar a esta población el pleno ejercicio del derecho a la educación en igualdad de oportunidades.

A esta corriente pertenecen esfuerzos como el "Programa de acción mundial para los impedidos" (11), la Declaración Mundial sobre Educación para todos y el Marco de Acción para satisfacer las necesidades básicas de aprendizaje (12); la Declaración hacia la plena integración de las personas con discapacidad: un programa de acción permanente (13) y las Normas Uniformes para la igualdad de oportunidades para las personas con discapacidad (14) (15) (6).

Posteriormente, instrumentos como la Declaración de Salamanca (16), la Declaración Mundial sobre la Educación Superior en el Siglo XXI (17) y el Marco de Acción de Dakar (18) avanzaron en la búsqueda de lograr que el acceso a la educación superior de personas con discapacidad se amplíe de forma progresiva, especialmente en países con poco crecimiento económico (6), bajo el fundamento conceptual del Principio de Educación Inclusiva para todos (16).

Se resalta además el avance logrado por la misma población con discapacidad en la declaración de la "Convención Internacional de los Derechos Humanos de las personas con discapacidad"1 (19), espacio en el que se hizo evidente la necesidad de hacer exigible su participación en igualdad de oportunidades en el contexto de la educación superior.

Sin embargo, y a pesar de los múltiples instrumentos internacionales (Tabla No. 1) que abogan por la igualdad de oportunidades y por la inclusión social, económica, política y educativa de las personas con discapacidad, el cumplimiento de los objetivos establecidos en las agendas gubernamentales aún sigue siendo limitado.

La Convención fue aprobada en Colombia a través de la Ley 1346 de 2009. 


\section{Tabla 1. Principales Declaraciones, normas y resoluciones de Organización de las Naciones Unidas (ONU) RELACIONADAS CON LA INCLUSIÓN EDUCATIVA DE PERSONAS CON DISCAPACIDAD.}

\begin{tabular}{|c|c|}
\hline Documento & Año \\
\hline Declaración universal de los derechos humanos. & 1948 \\
\hline Convención relativa a la lucha contra las discriminaciones en la esfera de & \\
\hline la enseñanza. & 1960 \\
\hline Pacto internacional de los derechos económicos, sociales y culturales. & 1966 \\
\hline Declaración de los derechos de las personas con discapacidad. & 1975 \\
\hline Programa de acción mundial para los impedidos se establece el período 1983-1992 como & \\
\hline Decenio de las Naciones Unidas para los impedidos. & 1982 \\
\hline $\begin{array}{l}\text { Protocolo adicional a la convención americana sobre los } \\
\text { derechos humanos en materia de derechos económicos, sociales }\end{array}$ & \\
\hline y culturales. OEA. & 1988 \\
\hline $\begin{array}{l}\text { Declaración mundial sobre educación para todos: la satisfacción de } \\
\text { las necesidades básicas de aprendizaje. }\end{array}$ & 1990 \\
\hline Declaración de Cartagena de Indias sobre políticas integrales para & \\
\hline las personas con discapacidad en el área iberoamericana. & 1992 \\
\hline $\begin{array}{l}\text { Declaración Hacia la plena integración en la sociedad de personas } \\
\text { con discapacidad: un programa de acción mundial permanente. }\end{array}$ & 1992 \\
\hline $\begin{array}{l}\text { Declaraciones: Inclusión plena y positiva de las personas con discapacidad } \\
\text { en todos los aspectos de la sociedad. Hacia la plena integración en la } \\
\text { sociedad de personas con discapacidad: un programa de acción } \\
\text { mundial permanente. }\end{array}$ & 1993 \\
\hline $\begin{array}{l}\text { Normas uniformes sobre igualdad de oportunidades para las personas con discapacidad, 1993. Resolución } 4896 . \\
\text { Declaración de Salamanca y Marco de acción para las necesidades }\end{array}$ & 1993 \\
\hline educativas especiales: acceso y calidad, Salamanca, España. & 1994 \\
\hline $\begin{array}{l}\text { Resolución Hacia la plena integración de las personas con discapacidad en } \\
\text { la sociedad: aplicación de las normas uniformes sobre la joualdad }\end{array}$ & \\
\hline de oportunidades. & 1994 \\
\hline $\begin{array}{l}\text { Resolución Estrategia a largo plazo para promover la aplicación del } \\
\text { programa de acción mundial para los impedidos hasta el año } 2000 \text { y años subsiguientes. } \\
\text { Resoluciones sobre la aplicación del programa de acción mundial para }\end{array}$ & 1995 \\
\hline los impedidos: hacia una sociedad para todos en el siglo XXI & $\begin{array}{r}1997-1999- \\
2001-2003\end{array}$ \\
\hline Declaración Americana de los Derechos y Deberes del Hombre. OEA. & 1998 \\
\hline $\begin{array}{l}\text { Declaración mundial sobre la educación superior en el siglo XXI: Visión } \\
\text { y acción y marco de acción prioritaria para el cambio y desarrollo de }\end{array}$ & \\
\hline la educación superior. & 1998 \\
\hline Educación para todos en las Américas: Marco de acción regional. & 2000 \\
\hline Educación para todos: cumplir nuestros compromisos comunes, & \\
\hline Foro mundial sobre la educación, Dakar, Senegal. & 2000 \\
\hline $\begin{array}{l}\text { Resolución sobre el establecimiento de una convención internacional } \\
\text { amplia e integral para promover y proteger los derechos y la dignidad }\end{array}$ & \\
\hline de las personas con discapacidad. & 2002 \\
\hline Convención sobre los derechos de las personas con discapacidad. & 2006 \\
\hline $\begin{array}{l}\text { Protocolo facultativo de la Convención sobre los derechos de las } \\
\text { personas con discapacidad. }\end{array}$ & 2008 \\
\hline
\end{tabular}

En esta misma línea de promoción de derechos de las personas con discapacidad, la UNESCO (6) (8) plantea el concepto de inclusión educativa como "una educación para todos, que corresponde a un proceso de direccionamiento y respuesta a las necesidades diversas de los educandos, y promueve la participación en el aprendizaje y la re- ducción de la exclusión" en el entorno educativo, haciendo posible a todas las personas sin distinción, ejercer el derecho a la educación.

"La igualdad de oportunidades educativas supone que cada cual tenga la posibilidad de satisfacer los deseos de recibir una educación compatible 
con sus capacidades a fin de lograr la preparación más adecuada para alcanzar las metas que cada ser humano se proponga, en una sociedad determinada por la competencia creciente $-\mathrm{y}$ a veces despiadada, en la cual el éxito material y deslumbrante termina por desplazar elementales exigencias de solidaridad, como la búsqueda de un sentido en el aprontamiento para vivir una existencia digna del hombre" (20).

Esta igualdad definida en términos de inclusión no es simplemente una modificación o ajuste de una serie de elementos presentes en el proceso educativo (objetivos, materiales, actividades, etc.), sino que supone un cambio profundo de la estructura, funcionamiento y modelo pedagógico, con la finalidad de dar respuesta a todos los estudiantes, incluidos aquellos que presentan una discapacidad (21).

Así, "hablar de inclusión significa aludir a la concepción más actual de discapacidad, en la cual hay que identificar y modificar aspectos ambientales y la interacción del alumno en cada contexto, más que centrarse en aspectos personales del déficit. La inclusión alude a la necesidad de cambiar los sistemas de atención y apoyo no sólo en el ámbito individual sino también en el de las organizaciones y la sociedad en general" (22).

\section{Educación Superior y Discapacidad en América Latina}

El concepto de inclusión educativa en tanto que resalta el derecho a la educación, debe extenderse a todos los ciclos de formación incluida la educación superior. Muestra de ello es el trabajo que viene desarrollando el Instituto Internacional de la UNESCO para la Educación Superior en América Latina y el Caribe-IESALC, el cual recopila las experiencias de inclusión educativa identificando el estado del tema en la región en aras a propiciar el desarrollo de políticas regionales que promuevan un marco de acción para una educación superior incluyente.
Ya que la ESI en Colombia no dista del desarrollo alcanzado por varios de los países de América Latina y el Caribe, se procede a continuación a hacer una breve descripción del estado actual de la inclusión educativa en algunos países de la región, para posteriormente analizar el desarrollo nacional.

En la legislación de América Latina se aprecia la tendencia a reconocer que la inclusión de las personas con discapacidad es un tema de derechos humanos. De acuerdo con datos de Moreno (23), en un gran número de países de la región se han aprobado en los últimos diez años una Ley General de Discapacidad o instrumentos jurídicos similares que se centran en la igualdad y equiparación de oportunidades, así como se ha dispuesto de una instancia nacional coordinadora y reguladora de los aspectos relacionados con las personas con discapacidad.

En el año 2004 el IESALC presentó un informe sobre el estado de la atención educativa de las personas con discapacidad en la educación superior (23), para lo cual estableció seis criterios de evaluación que pretendían identificar:

1. Restricciones legales al acceso, la permanencia y el egreso de la población con discapacidad a la educación superior de acuerdo con la Clasificación Internacional del Funcionamiento, de la Discapacidad y la Salud (2).

2. Definición de discapacidad que prima en el país de acuerdo con las leyes vigentes.

3. Caracterización de la población con discapacidad en la Educación Superior.

4. Organizaciones dedicadas a la protección y promoción de los derechos de las personas con discapacidad en el país.

5. Políticas y programas de las IES dirigidos a la inclusión e integración de las personas con discapacidad.

6. Conclusiones.

En estos informes se evidencia que el panorama es bastante diverso respecto a la cantidad de 
experiencias existentes, pero a la vez expone las múltiples falencias que aún persisten en el proceso de inclusión. Los informes disponibles corresponden a Argentina, Bolivia, Brasil, Chile, Costa Rica, Cuba, Ecuador, Guatemala, Honduras, Panamá, Paraguay, Perú, República Dominicana, Uruguay y Venezuela (24).

Países como Argentina, Brasil, Ecuador y Venezuela cuentan con leyes y normativas específicas para la educación superior. Se destaca la existencia en Brasil de una resolución que establece la accesibilidad (en sentido amplio) de las personas con discapacidad auditiva, visual y física como requisito para la autorización, reconocimiento y acreditación de las IES e incorpora este aspecto en su Sistema Nacional de Evaluación de la Enseñanza Superior (SINAES) (24).

A pesar de que en la región se cuenta con normas a favor de las personas con discapacidad, prevalecen los aspectos referidos al acceso y se concede menor atención a las estrategias favorecedoras de la permanencia y el egreso, lo que requiere de atención urgente ya que aún existen diversos factores que limitan su cumplimiento. Ejemplo de esta situación es el caso de países como Argentina y Cuba donde existe un modelo normativo que incluye el tema de la discapacidad de forma muy general, pero que carece de normativas especificas sobre educación superior.

En Ecuador, por el contrario, se encuentra un marco normativo que cuenta con leyes dirigidas especialmente a problemáticas de inclusión con respecto a la educación superior. En otros países como Honduras y Paraguay puede decirse que el modelo normativo es bastante deficiente, pues aunque incluye leyes con respecto a la población con discapacidad, éstas carecen de un suficiente desarrollo y en ocasiones presentan desactualización y validez normativa. Por último, en Venezuela, se identifican lineamientos que buscan el establecimiento regulado de deberes y compromisos hacia las IES con respecto al ingreso y permanencia de la población con discapacidad.

En cuanto a la caracterización de la población con discapacidad en la Educación Superior, se encuentra que Argentina ha desarrollado una encuesta nacional sobre población con discapacidad. En Cuba hay 12.547 estudiantes en instituciones de educación superior, lo que representa el $3.42 \%$ del total de la población universitaria.

En países como Ecuador, Honduras y Venezuela no hay información actualizada y los datos referentes a la cantidad de estudiantes integrados a instituciones de educación superior son parciales. Mientras que otros países como Paraguay cuentan con una caracterización demográfica de los estudiantes con discapacidad muy completa y detallada.

Examinando políticas y programas de las IES dirigidos a la inclusión de las personas con discapacidad se encuentra que en países como Argentina y Venezuela se proporcionan becas nacionales para esta población. En Cuba se ha aumentado el número de sedes regionales de las instituciones con el fin de optimizar y facilitar el acceso a estudiantes con discapacidad. Mientras que en Ecuador y Honduras se han desarrollado proyectos nacionales con el objetivo de proporcionar asesoría, capacitación y sensibilización a las instituciones para un optimo acceso de estudiantes con discapacidad. Adicionalmente, en Honduras se encuentran casos en los que este tipo de reformas se han hecho por iniciativas particulares de las instituciones educativas.

Una red nacional de apoyo a estudiantes con discapacidad ha sido configurada en Venezuela junto con jornadas y encuentros nacionales sobre el tema. Adicional a esto, se ha desarrollado 
la capacitación sobre temas de discapacidad y educación a funcionarios del Ministerio de Educación y la conformación de sistemas de información sobre educación superior y de atención a estudiantes con discapacidad.

Como resultado de los informes presentados por los países, se llevó a cabo el Primer Seminario Regional sobre la Inclusión de las Personas con Discapacidad en la Educación Superior en América Latina y el Caribe, celebrado en Caracas el 12 y 13 de diciembre de 2005, en cuya declaración final) (25) se exhorta a los gobiernos y a las agencias de acreditación de la educación superior a tomar diferentes medidas a favor de las personas con discapacidad, y se propende por la instauración en las IES de normas afirmativas para el diseño y desarrollo de programas y compromisos permanentes que garanticen la inclusión de esta población en la educación superior (6).

Derivado de esta declaración, se han generado estrategias de acción conjuntas que continúan con estos desarrollos, como la Declaración de Caracas en el marco del II Encuentro de consejos de rectores, redes universitarias y entidades vinculadas a la educación superior de América Latina y el Caribe (26), en la cual se resuelve apoyar el desarrollo de los proyectos del IESALC relacionados con la educación superior, entre los cuales se fortalecen la diversidad y la diferencia. Asimismo, estas se ven fortalecidas por discusiones de alto impacto derivadas de la Conferencia regional de educación superior en América Latina y el Caribe - CRES, llevada a cabo en junio de 2008 y la Conferencia Mundial de Educación Superior - CMES celebrada en julio de 2009 en París, en las cuales se exige a los países dar respuesta a las demandas sociales por medio de políticas de equidad para incorporar de manera efectiva y con calidad a diversos grupos sociales que han sido tradicionalmente excluidos, como es el caso de las personas con discapacidad (27) (28).

Finalmente, se destaca la consolidación de la Red interuniversitaria, latinoamericana y del Cari- be sobre discapacidad y derechos humanos, creada con el fin de fortalecer los derechos de la población con discapacidad en el contexto de la educación superior (29), dentro de la cual participa activamente Colombia.

Una vez recorrido el panorama latinoamericano, puede decirse que los diversos esfuerzos alcanzados en los países de la región muestran cómo se ha dado una respuesta lenta, basada en la normatividad internacional y que depende del interés político que se logre en cada país respecto al tema. Así mismo, resulta evidente que el panorama presentado no dista mucho de la situación de la inclusión educativa en la educación superior en Colombia, la cual se presentaré en el siguiente apartado.

\section{Análisis del caso colombiano}

La Constitución Política de Colombia en 1991 (30) consagró el papel del Estado en la protección especial para los "grupos marginados o desventajados de la sociedad que, en razón a su situación suelen ver limitado el ejercicio y el goce efectivo de sus derechos fundamentales". De esta manera, la acción del Estado colombiano en materia de discapacidad debería desarrollarse bajo los preceptos y principios constitucionales y en respuesta a la preocupación internacional y regional sobre la igualdad de oportunidades para estas personas. Dichas acciones tienen que ver, entre otras cosas, con el desarrollo de un marco normativo amplio, que reconozca los derechos y determine deberes de los diferentes actores, junto con la ratificación de convenios internacionales $^{2}(31)$.

Ley 115 de 1994, Ley 324 de 1996, Ley 361 de 1997 Decreto $\mathrm{N}^{\circ} 2082$ de 1996, Decreto $\mathrm{N}^{\circ} 2369$ de 1997, Decreto $\mathrm{N}^{\circ} 3011$ de 1997, Decreto $N^{\circ} 672$ de 1998, Normas Uniformes sobre la igualdad de oportunidades para las personas con discapacidad (1993) y Convención interamericana para la eliminación de todas las formas de discriminación contra las personas con discapacidad (1999), entre otras. 
Colombia cuenta con una amplia normatividad en el tema de la discapacidad, lo cual sustenta la inclusión educativa y exige a las instituciones la implementación de acciones tendientes a acelerar los procesos inclusivos en la educación en todos los niveles, así como el mejoramiento de las prácticas académicas, que compensen la relativa ausencia de condiciones de equiparación superiores.

Así mismo, en el país se han generado lineamientos para el ingreso de la población con discapacidad a la educación básica y media, los cuales no contemplan la transición a la educación superior. Es evidente que a esta última llega un número muy reducido de personas con discapacidad, lo cual se debe entre otras razones, a la reciente implementación de estas políticas en educación básica y media, generadas a partir de la promulgación de la Ley 115 de 1994.

Esta realidad se constata según reportes del DANE (2005), por los altos niveles de exclusión a la educación a los que se ve enfrentada la población con discapacidad; pues, según estos reportes, el nivel educativo de los hogares que albergan a esta población es mucho menor (32).

Los datos de acceso a la educación superior muestran que el $1 \%$ de la población culmina sus estudios superiores de carácter técnico, tecnológico o profesional, mientras que el $0,1 \%$ realiza algún postgrado (32). Este ínfimo porcentaje evidencia, no sólo, el reducido acceso a la educación superior, sino también, las restricciones en el acceso a la educación que vive esta población en los ciclos de formación anteriores, especialmente en a la educación inicial.

En estas mismas cifras, se destaca un importante porcentaje de la población con discapacidad que no ha alcanzado ningún nivel educativo (33,3\%) (32). Al respecto, sobre un estimado de 475.409 personas registradas en Colombia en el periodo $2003-2007^{3}$, Gómez expone los preocupantes niveles de analfabetismo presentes en la población con discapacidad. Las cifras muestran un $41 \%$ de niños entre 12 y 17 años que no saben leer ni escribir, un $42 \%$ de personas entre 18 a 24 años y un $33 \%$ de personas entre 25 a 44 años. De igual forma, se destaca que el $29,1 \%$ de esta población tiene un nivel de básica primaria incompleta (33).

Estas cifras hacen evidente que el principal obstáculo tiene que ver con el acceso inicial que se va reduciendo notablemente, hasta que se minimizan en la transición entre la educación básica y la superior, revelando la existencia de barreras estructurales que restringen las oportunidades en el acceso al nivel universitario. Los datos del Registro muestran que los menores de 12 a 17 años que deberían estar asistiendo a un establecimiento educativo no lo hacen en un $44 \%$ y entre las personas de 18 a 24 años la inasistencia es de $82 \%$ (33).

Estudios desarrollados en Colombia, demuestran que aunque la intención de atender a esta población existe, estas iniciativas suelen ser esfuerzos aislados en las instituciones, más no una política nacional que cuente con los recursos y el apoyo del estado que se precisa (34) (35). Adicionalmente, la autonomía universitaria expresada en el artículo 28 de la Ley 30 de 1992 (36), se interpreta de manera errada como una excusa para dejar a criterio de cada institución educativa la decisión de implementar las adecuaciones necesarias para garantizar el acceso, la permanencia y la educación de calidad a la población en situación de discapacidad.

En contraposición con esta idea cabe resaltar que la reciente Declaración de Buenos Aires (noviembre 6 de 2009), firmada en representación de Colombia por los senadores Carlos Ferro Solanilla, Alexander López Maya e Iris Rocío

El instrumento de base es el Registro para la localización y caracterización de la población con discapacidad, diseñado por el DANE para identificar a la población con discapacidad en Colombia. 
González Recalde, declara que es necesario "reconocer el valor de la autonomía como un componente necesario de la vida universitaria, entendiendo que autonomía y compromiso social con rendición de cuentas son en nuestra época dos conceptos indisolubles, que se requieren mutuamente para alcanzar su sentido más acabado", esto es, no olvidar el compromiso social que tienen las instituciones educativas de garantizar el derecho a la educación de calidad para toda la población, independientemente de su situación (37).

A pesar de los múltiples esfuerzos alcanzados hasta el momento a nivel legislativo y político, aún no existe en Colombia una política educativa específica que propenda por la reivindicación del derecho a la educación de las personas con discapacidad en la educación superior. Se ha dicho que "un marco legal rico no necesariamente garantiza que las acciones se estén llevando a cabo en la realidad, como en el caso de Colombia" (35). En este sentido, puede decirse que la situación local no dista de la de muchos de los países de América Latina, en lo que se evidencia un amplio espectro normativo pero cuya aplicación se ve limitada debido a diversos factores (6).

El panorama reportado muestra una realidad que expresa la exclusión a la que se ha visto enfrentada la población con discapacidad. Como parte de la respuesta que han dado diversas or- ganizaciones internacionales y nacionales, ha entrado en Colombia un auge por el tema de la inclusión educativa, el cual se vislumbra como un desafío para las IES, las cuales han comenzado a discutir e identificar medidas que respondan a las necesidades de este colectivo que demanda de manera progresiva su ingreso a diferentes niveles de formación.

El Ministerio de Educación Nacional inició en el año 2006 un trabajo encaminado a generar lineamientos y acciones para definir una política pública hacia una educación superior inclusiva, por medio de mesas de trabajo, identificando el panorama general y específico de la población con discapacidad con respecto a su acceso, permanencia y graduación. Finalmente, este trabajo llevó al primer Foro Un camino hacia la educación superior inclusiva, llevado a cabo en Bogotá los días 13 y 14 de agosto de 2007, en el cual se identificó que, si bien las IES han iniciado acciones que faciliten la inclusión a la educación superior, aún falta un gran camino por recorrer (38).

Con el fin de identificar los desarrollos alcanzados por las universidades, se presenta a continuación la Tabla 2, en la cual se sintetizan los avances reportados hasta el año 2007 por las universidades seleccionadas como experiencias exitosas en el Foro Un camino hacia la educación superior inclusiva (39).

TABLA 2. EXPERIENCIAS SIGNIFICATIVAS DE INCLUSIÓN EDUCATIVA PRESENTADAS EN EL PRIMER FORO UN CAMINO HACIA LA EDUCACIÓN SUPERIOR INCLUSIVA, 2007

\begin{tabular}{c|l}
\hline IES & \multicolumn{1}{c}{ Acciones desarrolladas } \\
\hline $\begin{array}{c}\text { Universidad Pontificia } \\
\text { Bolivariana - Bucaramanga }\end{array}$ & $\begin{array}{l}\text { - Formación para la participación y convivencia e incorporación comunidad universitaria. } \\
\text { Programa de asesoría académica tutorías académicas, mejoramiento de las estrategias de enseñanza- } \\
\text { aprendizaje y asesorías a estudiantes y docentes. } \\
\text { Proma de Acompañamiento } \\
\text { Académico }\end{array}$ \\
$\begin{array}{c}\text { - Formación de la familia y de voluntarios al proyecto. } \\
\text { - Vinculación activa del estudiante con discapacidad en el Programa de Acompañamiento Académico } \\
\text { PAC y proyección interna y externa. }\end{array}$ \\
\hline
\end{tabular}




\begin{tabular}{|c|c|}
\hline IES & Acciones desarrolladas \\
\hline $\begin{array}{c}\text { Sistema de Inclusión } \\
\text { Educativa }\end{array}$ & $\begin{array}{l}\text { - Cualificación de proceso de admisión. } \\
\text { - Desarrollo de videos en Lengua de Señas Colombiana para personas sordas y gráficas en relieve para personas } \\
\text { invidentes. } \\
\text { - Reconocimiento del tema por parte de la comunidad universitaria, sensibilización hacia la discapacidad. } \\
\text { - Fortalecimiento con diferentes dependencias de la universidad y con instituciones aliadas. } \\
\text { - Acompañamiento en la permanencia de los estudiantes con discapacidad. } \\
\text { - Desarrollo de acciones relacionadas con el egreso efectivo. }\end{array}$ \\
\hline $\begin{array}{l}\text { Proyecto Maxi-válido - } \\
\text { Diseño inclusivo }\end{array}$ & $\begin{array}{l}\text { - Articulación institucional para la inclusión entre bienestar, la academia y la administración. } \\
\text { - Acciones para impactar en la comunidad universitaria. } \\
\text { - Alcances sobre el proyecto educativo de la universidad en sus tres funciones: docencia, investigación y servicio. } \\
\text { - Búsqueda de máxima identidad, accesibilidad, funcionalidad, usabilidad y seguridad diseñando productos, } \\
\text { comunicaciones y espacios construidos más usables. }\end{array}$ \\
\hline $\begin{array}{l}\text { Universidad de } \\
\text { Manizales }\end{array}$ & $\begin{array}{l}\text { - Reflexión sobre emergencia de nuevas subjetividades (sujetos políticos, profesionales, heterogéneos) y nuevos } \\
\text { espacios de conocimiento (contenidos-conocimientos, ubicación diferente del maestro en el aula y flexibilidad). } \\
\text { - Se enfatiza en la consideración del desarrollo humano como multidimensional; compresión del aprendizaje } \\
\text { como policrónico. } \\
\text { - La comprensión de la pedagogía como base de la transformación de las instituciones en torno de la aceptación } \\
\text { y atención de la diversidad. } \\
\text { - El papel social que tienen en torno a la formación de una cultura de la inclusión. }\end{array}$ \\
\hline $\begin{array}{c}\text { Universidad Pedagó } \\
\text { Nacional }\end{array}$ & $\begin{array}{l}\text { - Dimensión pedagógica, dimensión académico administrativa, de investigación, vida universitaria, proyección } \\
\text { social. } \\
\text { - Voluntad y respeto por parte de la universidad, compromiso con la diversidad, presencia permanente de la } \\
\text { comunidad sorda, apoyo familiar. } \\
\text { - Curso de preparación, presentación de pruebas específicas, evaluación de la LSC. } \\
\text { - Organización del servicio de interpretación, diseño de los ambientes comunicativo y pedagógico, sensibiliza- } \\
\text { ción a la comunidad universitaria. } \\
\text { - Seguimiento y acompañamiento académico, apoyo pedagógico y tutorial, definición de criterios para la adecua- } \\
\text { ción y ajuste de los planes de estudio. } \\
\text { - Bienestar universitario: psicología, salud, restaurante, seguimiento pedagógico y permanencia. }\end{array}$ \\
\hline & $\begin{array}{l}\text { - Tutorías académicas para invidentes. } \\
\text { - Asignación de becas por compensación, subsidios de alimentación y monitorías académicas, participación en } \\
\text { eventos deportivos para invidentes. } \\
\text { - Elaboración de textos sobre accesibilidad. } \\
\text { - Desarrollo de prácticas académicas. } \\
\text { - Organizar grupos universitarios encaminados a la reflexión curricular, técnica y tecnológica. } \\
\text { - Fomentar procesos de sensibilización. Capacitar los diferentes órganos administrativos y académicos } \\
\text { institucionales. }\end{array}$ \\
\hline $\begin{array}{l}\text { Universidad de } \\
\text { Antioquia }\end{array}$ & $\begin{array}{l}\text { - Diagnóstico, elaboración de propuesta e implementación de servicio de acceso a la información para limitados } \\
\text { visuales desde la Biblioteca Central. } \\
\text { - Programa de voluntarios "Préstame tus ojos". } \\
\text { - Realización de obras de accesibilidad física. } \\
\text { - Conformación de la Comisión de Inclusión. } \\
\text { - Cursos de refuerzo de competencias básicas, braille, ortografía. } \\
\text { - Creación de CECUDEA - Comité de Estudiantes Ciegos de la Universidad de } \\
\text { - Antioquia. } \\
\text { - Adaptación y mejoramiento continuo del examen de admisión de las personas con limitación visual. }\end{array}$ \\
\hline $\begin{array}{l}\text { Fundación Universitaria } \\
\text { Católica del Norte }\end{array}$ & $\begin{array}{l}\text { - Educación virtual inclusiva para personas con movilidad reducida, invidentes y sordos. } \\
\text { - El sistema de educación virtual es abierto, flexible y asincrónico, optimizado las TIC como mediación efectiva } \\
\text { para los procesos de enseñanza y aprendizaje. } \\
\text { - Existen facilitadores virtuales (docentes) especializados en educación virtual. } \\
\text { - Se cuenta con una plataforma educativa probada. }\end{array}$ \\
\hline $\begin{array}{c}\text { Universidad de San } \\
\text { Buenaventura - Sede } \\
\text { Bogotá }\end{array}$ & $\begin{array}{l}\text { - Inclusión de personas sordas. } \\
\text { - Se cuenta con el apoyo de intérpretes, tutores (pares) y docentes. } \\
\text { - Se han construido estrategias particulares para profundizar en el conocimiento de la población sorda y en la } \\
\text { búsqueda de la apropiación de conocimiento para la enseñanza aprendizaje de la lengua de señas como elemento } \\
\text { esencial de necesidad pedagógica de la población sorda. }\end{array}$ \\
\hline
\end{tabular}


Según la tabla presentada, los programas de más desarrollo en lo que a inclusión educativa se refiere, se encuentran en las IES públicas quienes tienen una estructura multidisciplinar y cuentan con el protagonismo de estudiantes, profesores y funcionarios con discapacidad con apoyo de la comunidad universitaria (6).

Las IES han iniciado esfuerzos por incorporar a la población generando soportes conceptuales desde las disciplinas que han impulsado la inclusión educativa; las experiencias muestran que más que responder a un lineamiento institucional, la inclusión muchas veces ha surgido de docentes interesados en el tema, o se genera como respuesta al caso de estudiantes que han ingresado y los cuales presentan unas demandas particulares de apoyo y acompañamiento a la universidad.

De otro lado, las IES han intentado articular los diferentes responsables e implicados en los procesos de transformación y respuesta efectiva, por medio de estrategias de sensibilización a la comunidad universitaria, la incorporación de los estudiantes y de los docentes a la identificación de acciones, el acompañamiento académico por docentes y por grupos de pares voluntarios, la incorporación de bienestar universitario, así como la adquisición de tecnología de apoyo. Estas y otras actividades han permitido ajustes desde los procesos de admisión, de manera especial se destacan las transformaciones llevadas a cabo por las universidades que cuentan con exámenes de admisión; en los procesos de permanencia para evitar la deserción de la población, y en algunos casos en el acompañamiento para la transición a la vida laboral.

Muchas de las experiencias son aún jóvenes, y aunque han avanzado de manera importante hacia el logro de una educación con calidad para la población con discapacidad, no obstante, siguen siendo pocas en comparación con las necesidades de acceso de la población.

Una vez señalados los alcances y limitaciones de la ESI en Colombia, en este último apartado se identifican las líneas de trabajo sobre las que es necesario avanzar para alcanzar el propósito de garantizar el derecho a la educación de calidad en el nivel superior para la población colombiana que se encuentra en situación de discapacidad.

\section{Retos para el logro de una educación su- perior inclusiva de calidad en Colombia}

En primera instancia, el camino de la inclusión implica asumir acciones políticas y operativas que fortalezcan el trabajo interinstitucional e intersectorial, esto es, asumir que los retos de un servicio educativo adecuado no se reducen a alcanzar coberturas universales; pues para ello se necesita además, hacer de la educación una experiencia significativa para las personas, y pertinente a los requerimientos del desarrollo en el marco de una sociedad equitativa y solidaria (40).

Las cifras reportadas muestran una realidad que expresa la exclusión a la que se ha visto enfrentada la población con discapacidad. Se sabe que la incidencia y las consecuencias de la discapacidad se presentan más en lugares con bajos ingresos, situación que está directamente relacionada con la pobreza que se vive en muchos de los países de la región; de hecho, es de amplio conocimiento que la discapacidad tiene una relación directa con la pobreza (41). Lo anterior quiere decir, que el ingreso de la población a la educación en sus diferentes ciclos y de manera especial a la educación superior, es vital para desarrollar la identidad de las personas con discapacidad como individuos socialmente reconocidos y como ciudadanos activos dentro de la dinámica de su entorno, restableciendo la tesis de los valores, derechos humanos y obligaciones compartidos por la mayoría, dando la oportunidad de salir del tradicional círculo de pobreza - exclusión (42).

Sin desconocer los esfuerzos que hasta el momento han logrado las instituciones de educación superior en el país, así como las instituciones gubernamentales que trabajan en pro de la inclusión educativa de la población en situación de 
discapacidad en Colombia, es preciso afirmar que aún queda un amplio camino por recorrer, pues hasta que la educación superior no se comprometa con el desarrollo de la nación y con el bienestar de sus pobladores, incluso aquellos menos favorecidos; y mientras no se logre la unidad y convergencia entre ésta y la educación básica, el desafío de universalización del derecho a la educación superior seguirá siendo un asunto pendiente tanto para la población en situación de discapacidad, como para las demás poblaciones vulnerables del país.

Para ello es necesario que la universidad comprenda que su compromiso pedagógico con la universalización del derecho a la educación va más allá de la cantidad de matrículas registradas, y que la calidad de la misma implica, entre otros aspectos, generar las adecuaciones pertinentes para las poblaciones especiales, en este caso, la población con discapacidad. Si el primer paso es de aceptación y de apoyo hacia ellos, la población definitivamente tendrá un mejor futuro y su perspectiva de vida será completamente distinta (43).

Más allá de la responsabilidad propia de las IES, se deben considerar los compromisos de la transición de los niveles de educación media y vocacional al superior, contando con la participación institucional y de la misma población en un esfuerzo por articular los niveles de formación y por permitir que se subsanen brechas comunes que se suele presentar y que son determinantes en el acceso y en la permanencia de la población en el nivel superior (44).

El Estado por su parte, debe formular una política nacional que busque ampliar la cobertura, mejorar la calidad y garantizar la equidad de la educación superior, asumiendo para ello, la inversión que sea necesaria. Dicha política debe propender por la acción conjunta de las instituciones del estado tales como el MEN, el ICFES y el ICETEX con el fin de apoyar a la población en situación de discapacidad que no tiene los recursos necesarios para acceder a la universidad. Al mismo tiempo, debe ofrecer incentivos a las instituciones de educación superior para que avancen en la tarea de acomodarse a las necesidades de esta población, bien sea estableciendo la inclusión educativa como una dimensión de los procesos de acreditación de las instituciones universitarias, u ofreciendo asesoría y acompañamiento en dicho proceso a través de instituciones como el INCI y el INSOR. Además, debe ser una preocupación del Estado, la necesidad de establecer mecanismos efectivos de información sobre oferta educativa y demanda laboral para la población con discapacidad interesada en ingresar a la educación superior, o que egresa de la misma (45).

El Estado, a través de sus instituciones especializadas debe realizar estudios de caracterización de la población con discapacidad que se encuentra en el nivel de educación superior, con el fin de obtener información sobre la situación actual de acceso, permanencia, egreso e inserción laboral de estas personas. Así mismo, como parte de las acciones afirmativas que le competen, es necesario que los exámenes de estado, tanto para el ingreso como para el egreso a la educación superior, sean accesibles y se ajusten a las necesidades particulares de esta población. Entre tanto, la comunidad de personas con discapacidad, sus familias y las instituciones que las representan deben asumir el papel esencial que tienen en este proceso, en tanto veedores de la puesta en marcha de estas políticas y programas (45).

Las instituciones educativas por su parte, deben abrir sus puertas a la población con discapacidad, y hacer visibles sus respuestas efectivas a las necesidades de esta población, a través de la implementación de modificaciones en términos de accesibilidad al ambiente físico, a la información y a la comunicación. De forma paralela, deben realizar adaptaciones metodológicas e implementar estrategias novedosas para la enseñanza de las diferentes áreas del conocimiento.

Por tal razón, se debe pensar en la ESI desde un contexto más amplio, que no implique sola- 
mente la vinculación de la persona en situación de discapacidad al sistema educativo regular sino también la transformación de los entornos académicos, asegurando las condiciones de equiparación y accesibilidad necesarias para el desarrollo integral de estas personas, lo que además implica, la participación de todos y cada uno de los ámbitos sociales: la familia, la escuela, la comunidad y el sector laboral. De otro lado, los avances por lograr, deben también surgir de un compromiso ciudadano por la discapacidad, lo que involucra a las organizaciones de base y a la población en general, así como requiere del logro de una conciencia "solidaria, tolerante y pluralista" (46)(47)(48).

\section{Referencias}

1. Oliver M. Understanding Disability. From theory to Practice. London: MacMillan Press; 1996.

2. Organización Mundial de la Salud. Clasificación internacional del funcionamiento de la discapacidad y de la salud CIF. Madrid: Ministerio de trabajo y asuntos sociales de España; 2001.

3. Tomasevsky K. Los derechos económicos, sociales y culturales: el derecho a la educación. Naciones Unidas: Consejo Económico y Social; 2004.

4. Ramos D. Discapacidad e inclusión en la educación superior de América Latina y el Caribe. Aproximaciones conceptuales y avances de incorporación en proyectos estructurantes del IESALC. Ponencia. Foro: Un camino hacia la educación superior inclusiva. Ministerio de Educación Nacional, Ag. 13 y 14; 2007

5. Booth T, Ainscow M. Guía para la evaluación y mejora de la educación inclusiva. Consorcio universitario para la educación inclusiva. Departamento de Psicología Evolutiva y de la Educación. Traducción al castellano UNESCO-Santiago. Oficina Regional de Educación de UNESCO para América Latina y el Caribe; 2002. Disponible en: http://www.pangea.org/acpeap/doc\%20pdf/ index.pdf
6. IESALC - UNESCO. Las personas con discapacidades: los excluidos de la educación superior. Seminario "Integración de las personas con discapacidad en la educación superior en República Dominicana" UNAPEC IESALC/UNESCO, SEESCYT- CARMJ, CONADIS. Santo Domingo. Mayo 6; 2005.

7. Sandoval M, López ML, Miquel E, Durán D, Giné C, Echeíta G. Index for inclusion. Una guía para la evaluación y mejora de la educación inclusiva. En: Contextos Educativos, $\mathrm{N}^{\circ}$ 5. p. 227-238; 2002.

8. UNESCO. Superar La exclusión mediante planteamientos integrales en la educación. Un desafío y una visión. Documento conceptual. Autor: París; 2003. Disponible en http://www.unesco.org/education/inclusive.

9. Sverdlick I, Ferrari P, Jaimovich, A. Desigualdad e inclusión en la educación superior. Un estudio comparado en cinco países de América Latina. Serie Ensayos e Investigaciones No 9 Buenos Aires, 2005 Programa de Políticas da Cor (PPCOR) Observatorio Latinoamericano de Políticas Educativas (OLPED).

10. Misas G. La educación superior en Colombia: análisis y estrategias para su desarrollo. Bogotá: Universidad Nacional de Colombia; 2004.

11. Organización de las Naciones Unidas. Programa de Acción mundial para los impedidos. Asamblea General. Trigésimo séptimo período de sesiones. Resolución 37/52; 1982.

12. UNESCO. Declaración Mundial sobre Educación para todos y el Marco de Acción para satisfacer las necesidades básicas de aprendizaje; 1990.

13. Organización de las Naciones Unidas. Declaración hacia la plena integración de las personas con discapacidad: un programa de acción permanente; 1992. Disponible en: http://www.educared.edu.pe/especial/doc/ ley/Res47_88.pdf

14. Organización de las Naciones Unidas. Normas uniformes sobre la igualdad de oportu- 
nidades para las personas con discapacidad. Asamblea General A/RES/48/96; 1993.

15. Vicepresidencia de la República (1996). Discapacidad y derecho. Santafé de Bogotá: Autor; 1996.

16. UNESCO. Declaración de Salamanca y su Marco de Acción sobre Necesidades Educativas Especiales; 1994.

17. Declaración Mundial sobre la Educación Superior en el siglo XXI: Visión y acción (1998). Conferencia mundial sobre la educación superior, 1998. Disponible en http:// www.unesco.org/education/educprog/wche/ declaration_spa.htm

18. UNESCO. Marco de Acción de Dakar. Educación para Todos: cumplir nuestros compromisos comunes. Adoptado en el Foro Mundial sobre la Educación Dakar (Senegal); 2000.

19. Organización de las Naciones Unidas. Convención de derechos de las personas con discapacidad; 2006.

20. Sentencia T064/93. Igualdad de oportunidades. Derecho a la educación. Derechos fundamentales. Magistrado Ponente Ciro Angarita Barón.

21. Universidad Complutense de Madrid. Cátedra Microsoft de accesibilidad a la educación. Glosario; 2009. Disponible en:

http://cmae.mat.ucm.es/cmae/recursos/documentos/guias-de-accesibilidad/guia-docentepara-una-ensenanza-virtual-accesible-1/ capitulos/glosario.

22. Echeita G, Verdugo MA. La Declaración de Salamanca sobre necesidades educativas especiales 10 años después. Valoración y prospectiva. Salamanca: Ediciones del INICO, Universidad de Salamanca; 2004. Disponible en: [http://sid.usal.es/ mostrarficha.asp_Q_ID_E_9045_A_fichero_E_8.1]

23. Moreno MT. Integración/inclusión de las personas con discapacidad en la educación superior. En Iesalc- Unesco Informe sobre la Educación Superior en América Latina y el Caribe 2000-2005. Caracas; 2006. Capítulo 10. Págs. $144-155$.
24. IESALC. Observatorio de Educación Superior; 2004. Disponible en: http:// www.iesalc.unesco.org.ve/

25. Primer Seminario regional sobre la inclusión de las personas con discapacidad en la educación superior en América Latina y el Caribe. Declaración final. Caracas, Dic. 12 y 13; 2005. Disponible en. http:// inclusion.udea.edu.co/contexto/externo/ Declaracionfinal.pdf

26. Segundo Encuentro de Consejos de Rectores, Redes Universitarias y entidades vinculadas a la Educación Superior de América Latina y el Caribe. Caracas, Mayo 4; 2007. Disponible en: http://www.udual.org/Anuncios/07/DeclaracionCaracas.pdf

27. Declaración de la Conferencia regional de la educación superior en América Latina y el Caribe - CRES, Cartagena de Indias, Jun 4 al 6; 2008. Documento multicopiado.

28. Comunicado final de la Conferencia mundial de educación superior - CMES, París, Jul 6 a 8; 2009. Disponible en: http:// w w w. i n t e c.e d u . d o / p d f / Comunicado\%20CMES\%202009.pdf

29. Red interuniversitaria Latinoamericana y del Caribe sobre discapacidad y derechos humanos (2005) Documento de Buenos Aires - Argentina. Abril 25; 2009. Disponible en: www.rionegro.com.ar/blog/invisibles/files/ redinteruniversitaria.doc

30. Constitución Política de Colombia (1991) Artículo 67. Disponible en http:// www.banrep.gov.co/regimen/resoluciones/ cp91.pdf.

31. Departamento Nacional de Planeación. Consejo Nacional de Política Económica y Social. Conpes Social 80. Colombia, 2004.

32. DANE Censo general de población de 2005. Disponible en: www.dane.gov.co

33. Gómez JC. Magnitud de la exclusión educativa de las personas en situación de discapacidad en Colombia. Trabajo de grado para optar al título de magíster en Discapacidad e Inclusión Social, Universidad Nacional de Colombia; 2007. 
34. Cuervo C. Hacia una política académica inclusiva en la universidad colombiana. Trabajo de grado para optar al título de Magíster en Discapacidad e Inclusión Socia, Universidad Nacional de Colombia; 2007

35. Molina, R. Investigación Educativa de los estudiantes con discapacidad de la Universidad Nacional de Colombia. Trabajo de grado para optar al título de Magíster en Discapacidad e Inclusión Social, Universidad Nacional de Colombia; 2006

36. Congreso de Colombia. Ley 30, por la cual se organiza el servicio público de la Educación Superior; 1992. Disponible en http:// www.cna.gov.co/ $1741 /$ articles 186370_ley_3092.pdf.

37. ASCUN, IESALC, UNICEF. Declaración de Buenos Aires. Encuentro de senadoras y senadores de América Latina y el Caribe: educación superior en debate, desafíos normativos y derecho social a una educación de calidad. Buenos Aires, Nov 5 y 6; 2009.

38. Ministerio de Educación Nacional, Colombia. Convocatoria para participar en el programa de innovación en Educación Superior para población con discapacidad; 2009.

Disponible en: http://www.mineducacion.gov.co/ 1621/article-196813.html

39. Ministerio de Educación Nacional, Colombia. Memorias Foro educación superior inclusiva; 2007. Disponible en: http:// www.mineducacion.gov.co/1621/article$\underline{163417 . h t m l}$

40. Ministerio de Educación Nacional, Colombia, Educación Superior por ciclos y competencias; 2007 En: Sistema de aseguramiento de la calidad en educación superior.

41. Dudzik P, Elwan A. Metts R. Disability in Latin American and the Caribbean: A review of static and inclusionary policies. Seminar: dialogue on development and inclusion, opportunities for people with disabilities. Santiago de Chile: Inter-American Development Bank; 2001.

42. Vargas, DR. Representaciones sociales de la discapacidad en los consejos locales de discapacidad de la ciudad de Bogotá. Trabajo de grado para optar al título de Magíster en Salud Pública, Universidad Nacional de Colombia; 2006

43. Fernández A., Díaz, OC. Problematización sobre las concepciones vigentes en la educación de las personas en situación de discapacidad. En: Cuervo C, Trujillo A, Vargas DR, Mena B, Pérez L. (Eds.) Discapacidad e Inclusión Social: Reflexiones desde la Universidad Nacional de Colombia; 2005.

44. Balcazar F. Opciones de la transición. Ponencia. Foro: Un camino hacia la educación superior inclusiva. Ministerio de Educación Nacional. Bogotá. Ag 13-14; 2007.

45. Flórez R., Moreno M., Bermúdez G., Cuervo G. Propuesta de lineamientos de política para la atención educativa de poblaciones en situación de discapacidad en las instituciones de educación superior en Colombia; 2009 Manuscrito no publicado. Bogotá.

46. Molina R. Opiniones, debates y controversias. Hacia una educación con igualdad de oportunidades para personas con discapacidad. En: Revista Faculta de Medicina, Universidad Nacional de Colombia,2006, 54,2.

47. Agenda de Políticas y Estrategias para la Educación Superior Colombiana 2002 - 2006. «De la Exclusión a la Equidad» Bogotá, D.C., mayo de 2002 http://www.cuib.org/ agenda_politicas_escolombiana.pdf

48. Ministerio de Educación Nacional, Colombia. Boletín Digital de Educación Superior No.59 - Miércoles, 21 de Noviembre, 2007. 